\title{
Cerebral revascularization: direct versus indirect bypass. Case presentation and review
}

\author{
A. Giovani, F. Brehar, R.M. Gorgan \\ “Bagdasar Arseni” University Hospital, Bucharest
}

\begin{abstract}
Since 1985 when the EC-IC bypass study results were published and less procedures were performed for cerebral ischemia, the EC-IC bypass even high or low flow and the IC-IC bypass as flow replacement procedures gain acceptance for many neurosurgical pathologies, from extrinsic and intrinsic tumors requiring large vessels sacrifice to large giant and fusiform aneurysms. In recent years, after the results of Carotid Occlusion Surgery Study (COSS) and the Japanese EC-IC trial published their results the indications for extracranial-intracranial (EC-IC) by-pass expanded, including both extracranial carotid artery occlusive disease and intracranial atherosclerotic disease. The authors make a literature review of the indications for cerebral revascularization, with focus on the direct STA-MCA and indirect (EDAMS) revascularization techniques as a treatment for ischemic stroke. They present two cases of Moyamoya disease one treated with combined approaches and one with indirect approach and discuss the technical skills the surgeon should acquire in order to perform an anastomosis, focusing on the details of STA-MCA bypass, concluding that a combined approach gives better neurological results visible shortly after the surgery.
\end{abstract}

Key words: cerebral revascularization, extracranial-intracranial by-pass, stroke.

\section{Introduction}

Cerebral revascularization either surgical through very technical demanding bypasses or endovascular through pipeline embolisation devices, stenting or trombectomy devices refers to bringing cerebral perfusion in teritories where it is lacking due to vessel occlusion or where it will be lacking after the vessels involved in a large extra/intraaxial tumor or giant aneurysm will be sacrificed during the treatment of that specific condition $(3,7)$. In the first case we speak of a flow augmentation bypass while in the second it is a flow replacement bypass. For flow augmentation in the anterior circulation the most used bypass is a low-flow bypass, the superficial temporal artery (STA) to middle cerebral artery (MCA), while for the posterior circulation occipital artery (OA) to posterior inferior cerebellar artery (PICA) or STA to superior cerebellar artery (STA) are the 
alternatives. In cases of giant or complex aneurisms and skull base tumors where flow replacement is mandatory a high flow bypass is appropriate to replace the flow through the sacrificed vessel. Even if the patient does not fail the balloon occlusion test there is a $30 \%$ risk of subsequent ischemia and stroke. Different types of bypasses have been described, from direct intra-intracranian (ICIC) like termino-terminal, reimplantation or laterolateral to IC-IC using short grafts like petrous carotid to MCA or internal maxillary artery to MCA, or IC-EC bypasses using long radial or safenous grafts to take blood from the common carotid artery to the middle or posterior cerebral artery (14).

Preoperative flow measurement is not readily available in many departments ours included which lack Xenon CT, perfusion CT, perfusion MRI, spect, transcrannial Doppler or PET so in this centers the decision for the revascularization intervention is based only on the clinical and angiographic criteria.

The normal cerebral blood flow $(\mathrm{CBF})$ is 46 $\mathrm{ml} / 100 \mathrm{~g} / \mathrm{min}$ and oxygen extraction fraction (OEF) is $30 / 40 \%$ so the oxygen supply is two to three times greater than oxygen demand. The Kyoto and St. Louis trials based on hemodynamic criteria demonstrated that in stage II hemodynamic failure defined as increased OEF as measured by PET studies, the medical treatment results were poor and comparable to those reported for symptomatic severe carotid stenosis $(12,13)$. As the EC-IC bypass is known to normalize the OEF in patients with increased OEF distal to an occluded carotid artery it should be the treatment of choice for this subset of patients (2).
The JET study measured the CBF and the reduced acetazolamide cerebrovascular reactivity with SPECT while the COSS trial includes for eligibility Clinical Criteria, PET scan and arteriographic criteria, including angiography.

After the patient has been included for the treatment a last line of physiological measurments must follow: the intraoperative flow measurement. This technique allows the surgeon to pick the appropriate STA branch for by pass and to check immediately the patency of the anastomosis. Postoperative the state of the flow through the anastomosis should be checked by quantitative MR Angiography.

Creating a bypass is very demanding technically and many days should be spent acquiring the required skill in the lab under the highest magnification of the microscope.

\section{Lab training}

No in vivo anastomosis should be performed without previous intensive lab training. It is advised to use first swine heart coronary arteries than smaller chicken wing and only when comfortable with these the next step, anastomosis in anesthetized mice first on carotid than on inguinal arteries should be performed. All kinds of anastmosis, starting with termino-terminal (TT), than latero-lateral (L-L) termino-lateral (T-L) should be exercised. (Figure 1 and Figure 4) One must try to view any anastomosis as a L-L one as this concept allows the surgeon to perform TT and TL anastomosis in deep locations working in narrow corridors, without the need to rotate the anastomosis in order to make the second anastomotic line 
DOI: 10.2478/romneu-2014-0062

visible. In the begging using separate $10-0$ or 11-0 sutures is advised by most of the experts but one may find the continuous technique faster and easier. When the trainee feels he acquired enough experience he/she must use a continuous perfusion system and clips to make it as close as possible to in vivo conditions and only than anastomosis on anesthetized mice and then on humans can be performed. (Figure 2 and Figure 3). No high flow anastomosis should be tried unless dozens of low flow ones were previously done with success. A good exercise is to construct simulated aneurysms for clipping training as in video:

https://www.youtube.com/watch?v=H6h4 NKbbHkk\&index $=5 \&$ list $=$ UUAC_q8QnPMd YQwbc6x_63WA

https://www.youtube.com/watch?v=L4NB pcDg39c\&list=UUAC q8QnPMdYQwbc6x 63WA\&index $=3$

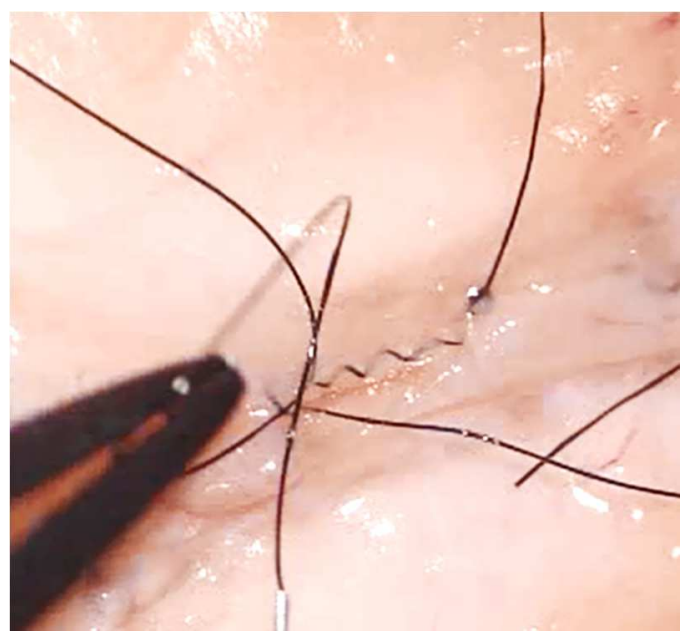

Figure 1 - Latero-lateral anastomosis on chicken wing arteries - the back wall was sutured and the suture is continued on the front wall

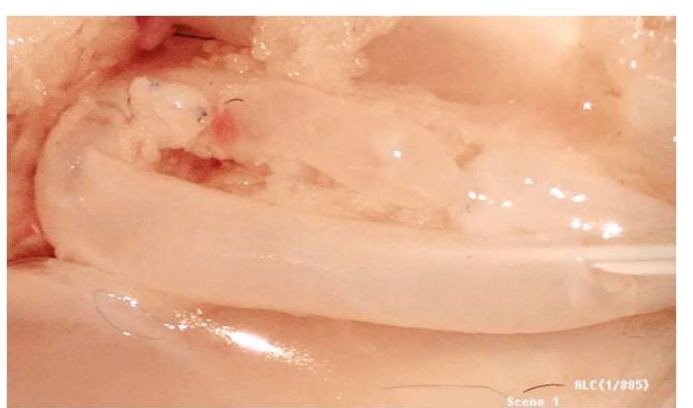

Figure 2 - a small aneurysm (black arrow) was reconstructed from a segment a vein. The aneurysm fills as the vessel is irrigated

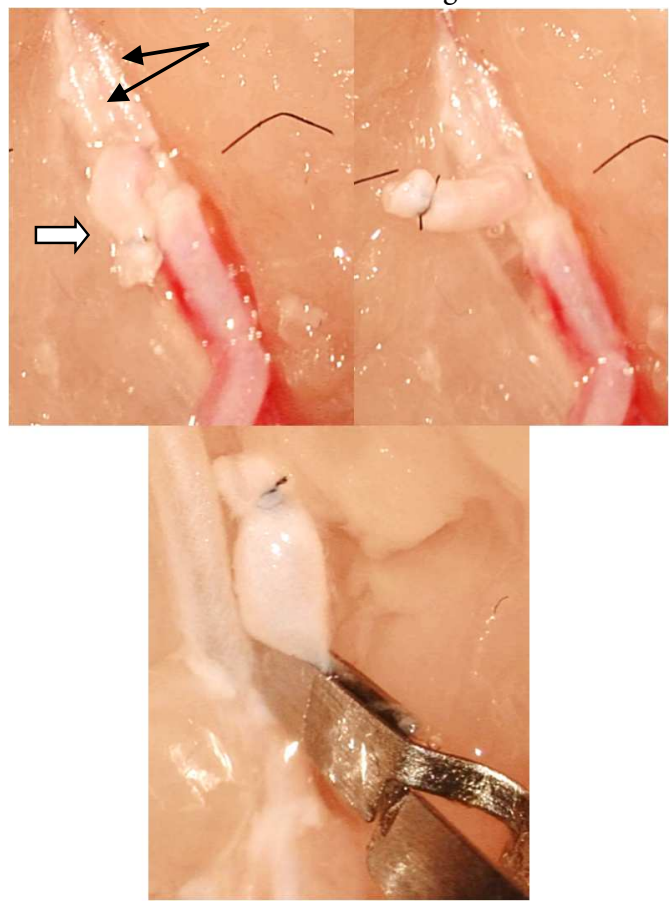

Figure 3 - a segment of an artery closed in one end is anastomosed at an artery bifurcation (black arrows) creating an aneurysm (white arrow) that is filled with water from the continuous infusion system and then clipped 


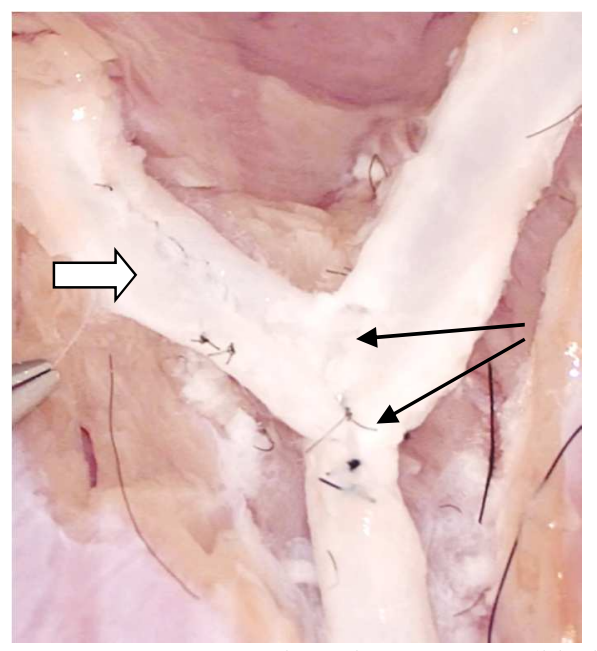

Figure 4 - Two terminolateral anastomosis (black arrows); the two vessels are united by a laterolateral anastomose (white arrow) and the vessels are filled with water from the continuous infusion system showing no leakage

\section{STA-MCA bypass}

One of The best indications for STA-MCA bypass is moyamoya disease, yet the by-pass should be considered only in cases with lack of response to maximal medical treatment.

The cerebral flow should be investigated preoperative using CT perfusion or Xenon CT blood flow measurement or PET for oxygen extraction measurement. Than the feasibility of the bypass should be evaluated measuring the caliber of the STA on a 6 vessels angiography.

Every step in performing an Anastomosis is very important and any mistake can compromise the whole procedure.

According to the team's preferences the head can be fixed in Mayfield or left free on horseshoe with the head parallel to the floor. After identifying and mashin marking the STA and its branches by palpation or using a
Doppler probe a vertical Linear incision in front of the ear is used with care not to injure the vessel. Use of the operating microscope from the skin incision is advisable. During dissection small branches should be coagulated while lager ones should be ligated and cut.

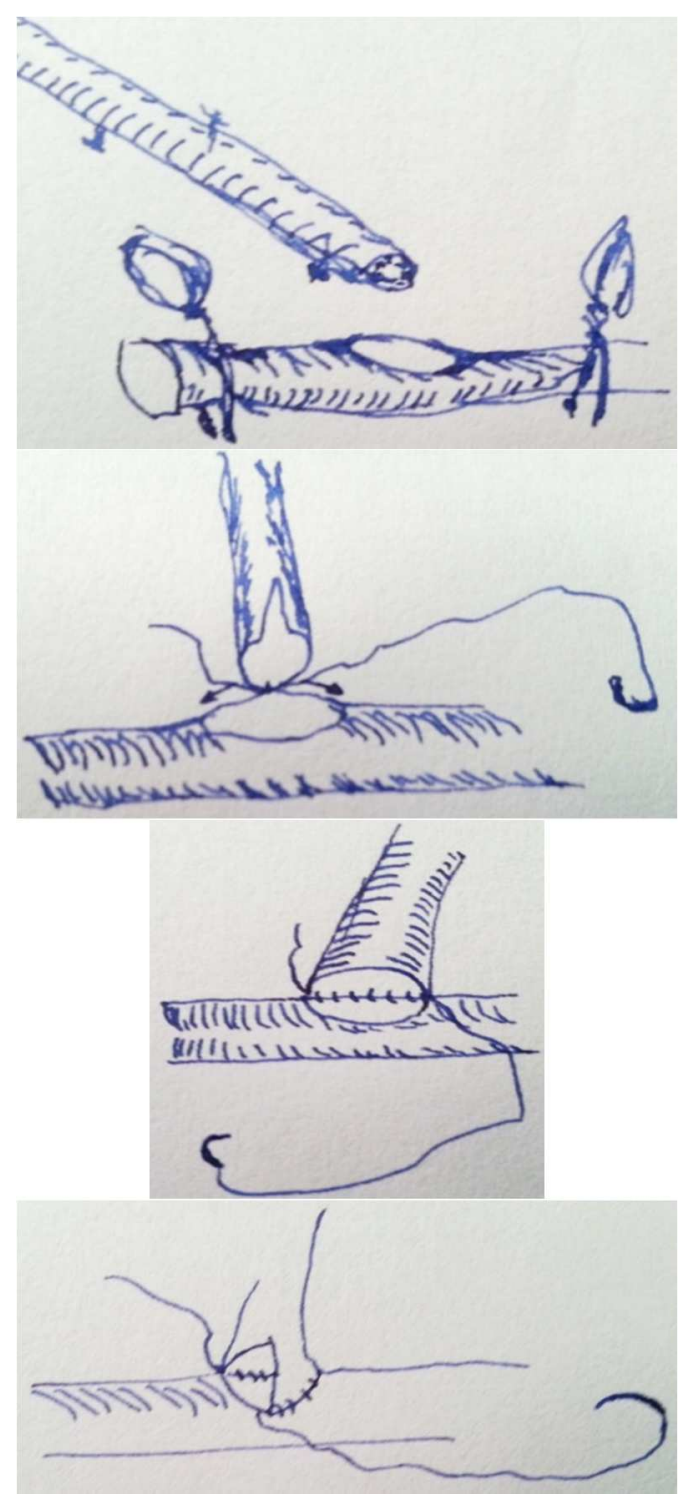


DOI: 10.2478/romneu-2014-0062

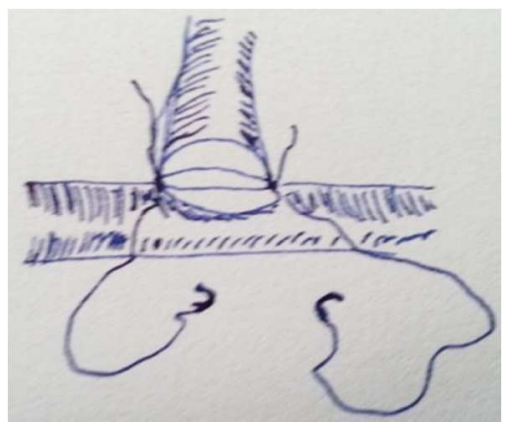

Figure 5 - Two ways of performing a termino-lateral anastomosis, using one thread that is sutured to its own tail in the end or two heel stiches that go continuously and are sutured to each other's tail in the end

The STA should not be completely denuded during dissection as this may cause injury. When this step is completed the artery is retracted and fixated posteriorly in a papaverine soaked cottonoid. Then the temporal muscle is split with the cautery on the midline and desinserted from the bone. A $3 / 3 \mathrm{~cm}$ diameter craniectomy is performed and the dura is incised. After finding a proper caliber $\mathrm{m} 3$ or $\mathrm{m} 4$ vessel the STA is clipped proximally and cut as to assure a propper length for the Anastomosis not to be in tension. At this step if a Charbel Doppler probe is available it should be used to measure the cut flow index. The distal end of the STA is fish mouthed to increase the diameter of the Anastomosis. Next a proximal $3 \mathrm{~mm}$ clip should be placed on $\mathrm{m} 3$ or $\mathrm{m} 4$ followed by a distal clip. During this maneuver the blood pressure should be increased. The distance between the clips must be at least $1 \mathrm{~cm}$ to allow a good working space between the clips. Then a linear incision is made along the axis of the $\mathrm{m} 3 / \mathrm{m} 4$ vessel using a needle tip to make a puncture and a strait or angulated scissors to complete a 2,5 $\mathrm{mm}$ incusion (more than twice the diameter of the receiving vessel). The vessel must be irrigated with heparinised saline and some authors prefer a colored dye to see the margins. Two sutures are placed at 0 and 180 degrees (heel and toe) and then the suture lines are runned continuously with 100 , placing the stich as close to the edge as possible. The back wall is completed first and then the front, more proximal suture line. The Anastomosis is continuously irrigated till the final closure. The distal clip is removed first than the proximal than the clip on STA. (Fig 5) A little ooze from the Anastomosis line is normal. If there is true bleeding the clips must be reapplied and another stich should close the opening. After the completion of the anastomosis the Charbel Doppler probe is used again to check the flow through the Anastomosis. If the cut flow index is less than 0,5 it means there is a technical problem with the bypass and It may need revision.

The next step of the surgery is applying the temporal muscle intimate on the pial surface with care not to occlude the STA coming between its two halves. The two layers are approximated to each other on the midline and to the suspended dura on the edges.

\section{Case 1. Combined direct/indirect approach}

A 32 yo male presented with Left mild hemiparesis and Dysarthria that worsened over the week prior to admission. A CT scan showed marked hypodensity areas in the right frontal and parietal lobes in the territory irrigated by right MCA. (Figure 6) The almost complete carotid occlusion was observed on the right internal carotid artery angiography 
and very few basal collaterals which corresponded to a grade VI Moyamoya disease on Suzuky classification. The patient opted for a combined direct-indirect approach. The surgical technique was described above. A video with the technique can be accessed here: https://www.youtube.com/watch?v=d AdljV o0-Q\&list=UUAC q8QnPMdYQwbc6x 63WA;

https://www.youtube.com/watch?v=u60ZURJ

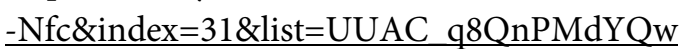
bc6x_63WA.

The patient received heparine 1 day before and one day after the surgery and then was discharged on chronic Aspirin treatment. Given the small caliber of the anastomosed vessels hypotension should be avoided in the first postoperative days to maintain a good flow through the bypass. (Figure 7)

The outcome was favorable. Dyasrthira and left hand deficit improved in the first postoperative week and the patient was able to Ambulate without help at discharge after 1 week. At two months follow up the patient was able to hold his 4 yo daughter in his left hand, and he presented no speech impediment. (Figure 8)

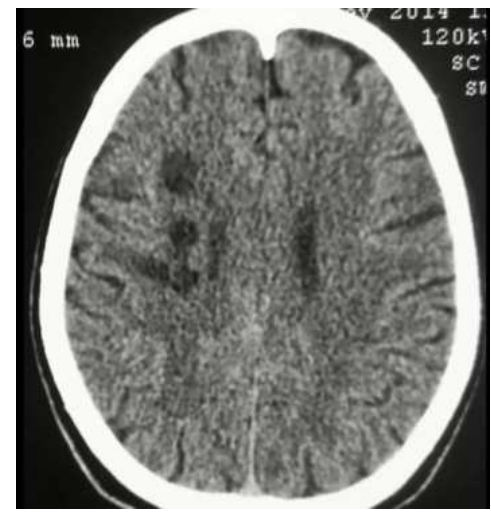

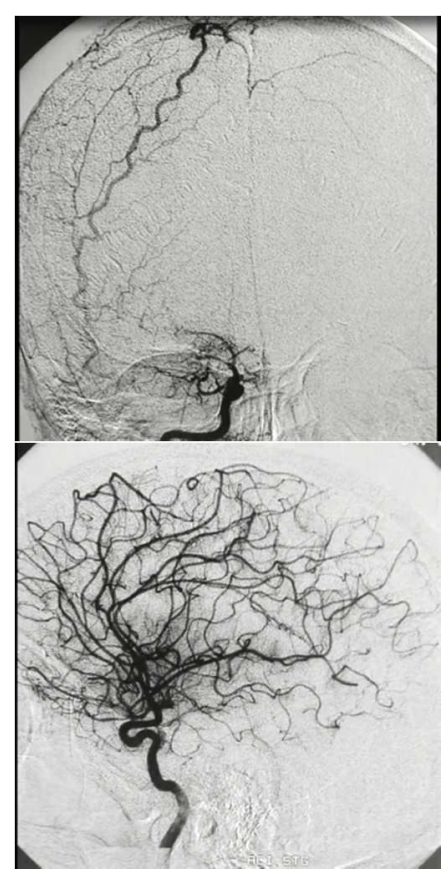

Figure 6 - CT scan and angiography showing ischemia and complete intracranial ICA occlusion corresponding to a grade VI Moyamoya on Suzuky classification

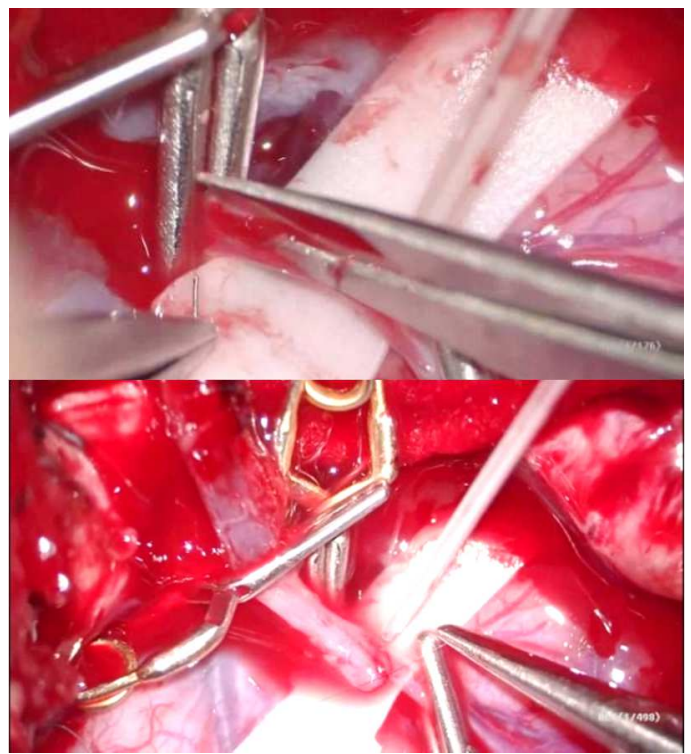


DOI: 10.2478/romneu-2014-0062

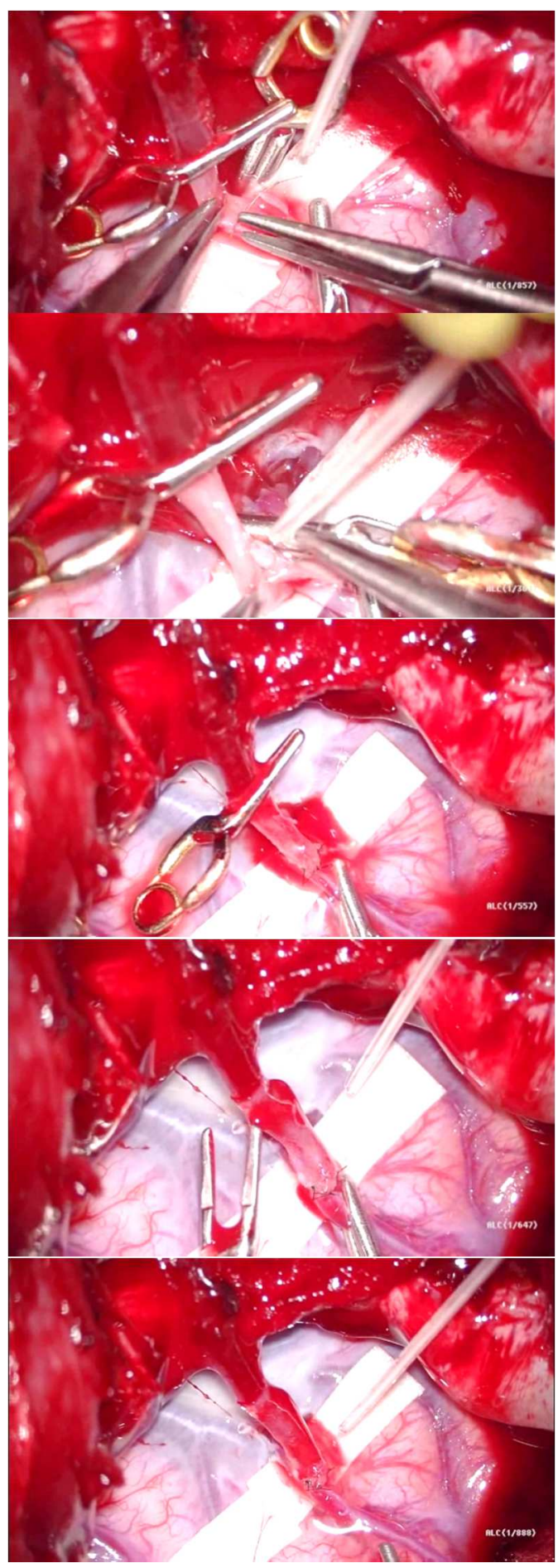

Figure 7 - intraoperative pictures with the technique (also see video) showing the linear cut in the recipient vessel, the first heel stich, and after the completion of the anastomosis the removal of the clips, in this case first the proximal one on the recipient vessel, note a little ooze from the anastomotic line and then note the increase in size of recipient vessel between the anastomosis place and the distal clip when the clip on the STA (black arrow) is removed, and the pulsating vessel with no leakage or stenosis after removal of the last clip

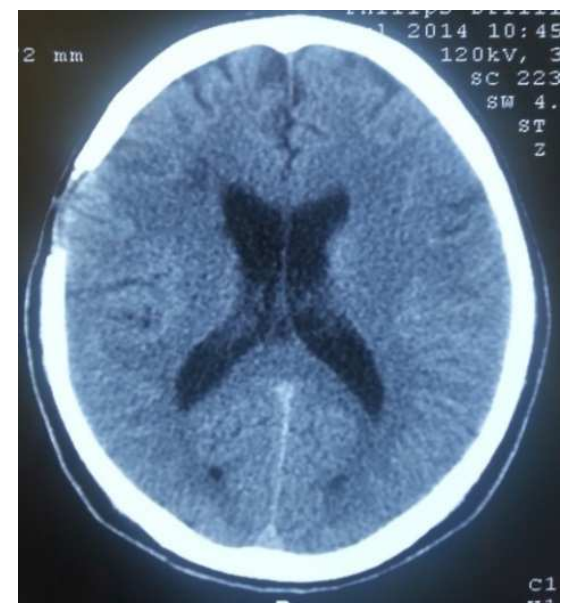

2 months CT scan

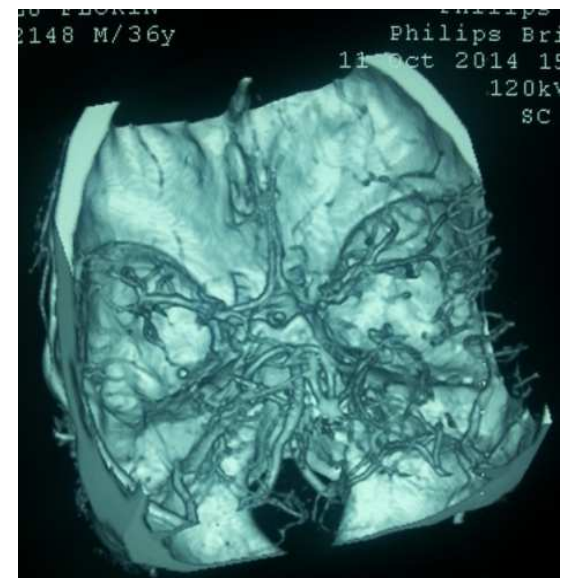

6 months Angio CT

Figure 8 - Postop CT scan at two months shows no hypodensity in the territory of the right MCA and the 6 months follow up angio CT shows many collaterals formed in the right fronto temporo parietal area 


\section{Case 2}

\section{Encephaloarterioduromyosynagniosis (EDAMS)}

A 36 years old woman presented with motor aphasia, Left hemiparesis predominant in the arm, Left central facial nerve paresis, Facial paresthesia and Visual deficit. The symptoms appeared and worsened over the last 2 weeks.

The MRI showed multiple ischemic frontal and parietal lesions, note IVI sign on the pial surface and the flow void signals given by the basal ganglia collaterals.

Angiography showed bilateral occlusion of the intracranial carotid artery with collaterals formation corresponding to a Suzuky grade IV. (Figure 9)

The chosen treatment was bilateral Encephaloarterioduromyosynagniosis (EDAMS).

Using a linear temporal skin incision the STA was dissected from the surrounding tissue and preserved in continuity. A $3 / 3$ temporal craniectomy was performed after the mobilization of the temporal muscle with care not to injure its vascular pedicle. The dura was opened and suspended circullary to the fascia. The arachnoid was opened over the cortical surface and the STA and then the temporalis muscle were applied on the pial surface. The muscle is then fixed in place with a few interrupted stiches to the reflected dura, and the skin was closed. (Figure 10)

At 6 months follow up the hemiparesis and aphasia recovered completely and the 7th nerve paresis recovered incompletely (Figure 11).

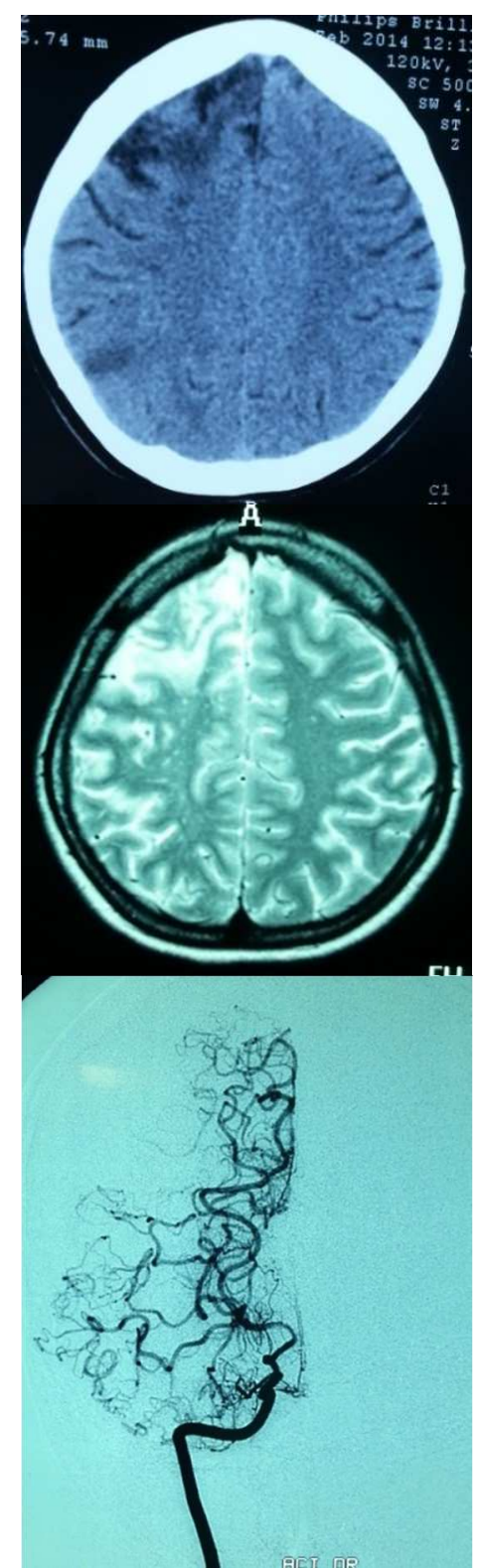

Figure 9 - Preop CT and MRI showing right frontal ischemia, and pial collaterals on T2 called IVI sign. The angiography shows an amputated flow in the MCA and formation of basal collaterals, consistent with a Suzuky 4 grade 
DOI: 10.2478/romneu-2014-0062
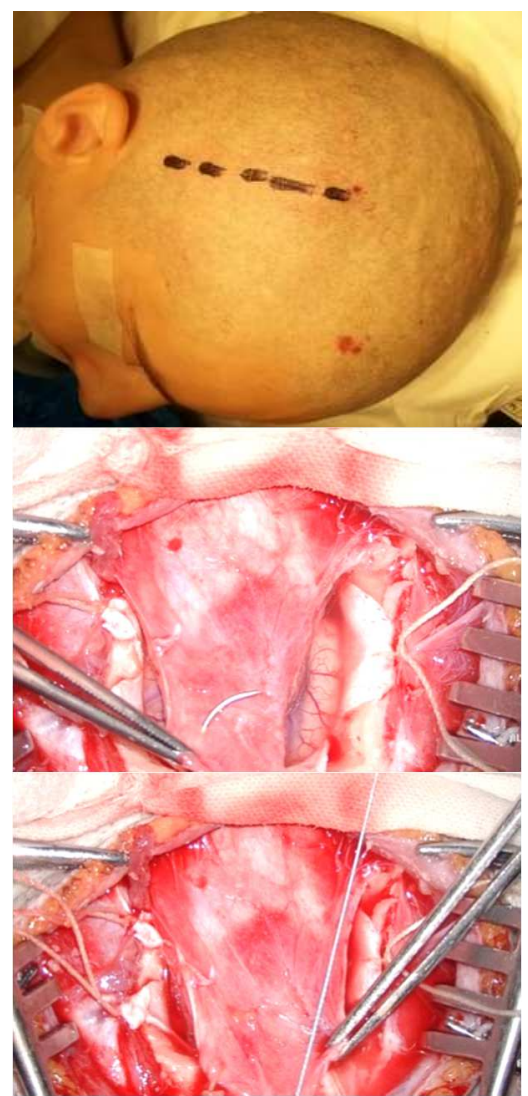

Figure 10 - Intraoperative images showing the technique

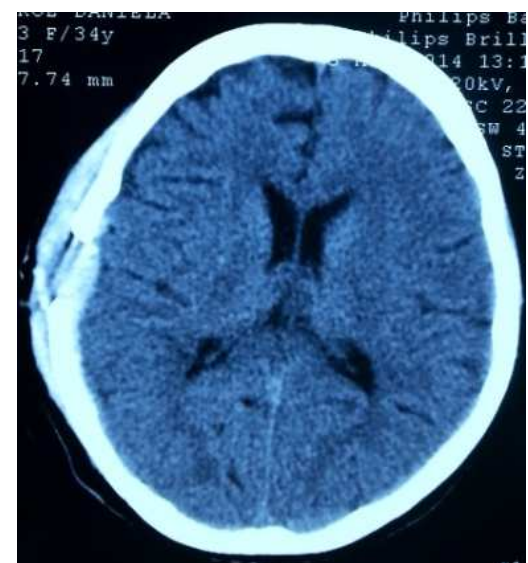

Figure 11 - The postop CT shows the disappearance of frontal hypodensity at 6 months follow up

\section{Discussion}

Since 1985 when the EC-IC bypass study results were published and less procedures were performed for cerebral ischemia, the ECIC bypass even high or low flow and the IC-IC bypass as flow replacement procedures gain acceptance for many neurosurgical pathologies, from extrinsic and intrinsic tumors requiring large vessels sacrifice to large giant and fussiform aneurysms (3).

In recent years, after the results of Carotid Occlusion Surgery Study (COSS) and the Japanese EC-IC trial published their results the indications for extracranial-intracranial (ECIC) by-pass expanded, including both extracranial carotid artery occlusive disease and intracranial atherosclerotic disease (7).

As $10 \%$ of TIA and $15-25 \%$ of ischemic strokes are caused by complete carotid artery occlusion and under the best medical treatment the two years risk of subsequent stroke is of $10 \%-15 \%$ surgical revascularization was reconsidered in diminishing this risk in many cerebrovascular centers. Intracranial atherosclerotic disease accounts for $9 \%$ of all ischemic strokes and has a 2 years recurrence rates of $15 \%-20 \%(8,9)$.

Even if Coss trial didn't show clear evidence in favor to surgery the postoperative ischemic strokes (14/93) cannot be attributed to technical problems with the bypass, but to patient hemodynamic fragility due to age and inability of patients to tolerate surgery.

Thus decision making based on preoperative and intraoperative flow measurement is very important when selecting 
patients for by-pass, even direct indirect or combined.

Large metaanalysis showed that in one third of the cases the STA-MCA anastomosis is combined with miosynangiosis with a growing tendency for this technique. When combined direct and indirect approach is used, it was shown that the bypass provides the patient with a immediate increase in blood flow and decrease of OEF, translated in a recovery of the neurological symptoms, but in time, after the first 6 months postoperative the collaterals that form form the miosynangiosis take over the workload of the anastomosis and when this is checked with a QMRI it will show a severe decreased flow through the anastomosis compared to the same measurement done in the first weeks after the surgery $(1,10)$.

Conflicting conclusions are found in different studies, some performed only on patients with indirect bypass criticize the STAMCA as prone to induce thrombosis at the place of stenosis and this thrombosis can occlude the lenticulostriate arteries giving rise to new neurologic deficit, and advocate the use of indirect bypass alone, either encephaloduroarteriosynangiosis (EDAS) or enecephaloduromioarteriosynangiosis

(EDAMS) (5). Other studies performed on large numbers of patients who benefited from both direct and indirect approach show very good results in the rate of postoperative stroke (4.7\%) combined to medical treatment or to indirect approach alone with even better results in pediatric compared to adult population $(4,6)$.
In our poor experience with these techniques we noticed that the neurological recovery after the combined approach is better and comes more rapidly than with myosinangiosis alone.

\section{Conclusions}

The refinement of anesthetic methods allows expert vascular neurosurgeons to operate with a high rate of success complex cases of giant aneurysms and tumors previously considered impossible, now even in awake setting. After a decline since 1985 revascularization procedures are reconsidered for cerebral ischemia.

A combined direct - indirect approach is better than using each technique alone, for patients with simptomatic extracranial or intracranial carotid occlusion given the fact that STA-MCA bypass provides early augmentation of flow and EDAMS provides durable long term revascularization and one of the best indications for the technique is Moyamoya Disease.

Every high volume center should have a few vascular neurosurgeons very well trained in microanastomosis techniques.

No bypass procedure should be performed without a good indication, yet Moyamoya disease when symptomatic represents one of the best indications.

\section{Correspondence}

Andrei Giovani

giovani.andrei@gmail.com 
DOI: 10.2478/romneu-2014-0062

\section{References}

1. Amin-Hanjani S, Shin J, Zhao M, et al : Evaluation of extracranial-intracranial bypass using quantitative magnetic resonance angiography J Neurosurg 106: 291298, 2007

2. Amin Hanjani S, Charbel FT: Is extracranialintracranial bypass surgery effective in certain patients? Neurol Clin 24: 729-743; 2006.

3. ARTHUR L. DAY, M.D. ; Indications for surgical intervention in middle cerebral artery obstruction ; Neurosurg 60:296-304, 1984

4. James A. J. King, M.B., B.S., Ph.D., F.R.A.C.S.,1 Derek Armstrong, M.B., B.S., F.R.C.P.,2 Shobhan Vachhrajani, M.D.,3 and Peter B. Dirk s, M.D., Ph.D., F.R.C.S.C3 ; Relative contributions of the middle meningeal artery and superficial temporal artery in revascularization surgery for moyamoya syndrome in children: the results of superselective angiography. ; J Neurosurg Pediatrics 5:000-000, 2010

5. Joshua R. Dusick, M.D.,1 David S. Liebeskind, M.D.,2,4 Jeffrey L. Saver, M.D.,2,4 Neil A. Martin, M.D.,1 and Nestor R. Gonzalez, M.D.; Indirect revascularization for nonmoyamoya intracranial arterial stenoses: clinical and angiographic outcomes; J Neurosurg 117:94-102, 2012

6. Ken Kazumata, M.D.,1 Masaki Ito, M.D.,1 Kikutaro Tokairin, M.D.,1 Yasuhiro Ito, M.D.,1 Kiyohiro Houkin, M.D.,1 Naoki Nakayama, M.D.,1 Satoshi Kuroda, M.D.,2 Tatsuya Ishikawa, M.D.,3 and Hiroyasu Kamiyama, M.D.; The frequency of postoperative stroke in moyamoya disease following combined revascularization: a single-university series and systematic review; J Neurosurg 121:432-440, 2014

7. Maxim Mokin, M.D., Ph.D.,1,2 Peter Kan, M.D., M.P.H.,3,4 Tareq Kass-Hout, M.D.,1,2 Adib A. Abla, M.D.,3,4 Travis M. Dumont, M.D.,3,4 Kenneth V. Snyder, M.D., Ph.D.,3-6 L. Nelson Hopkins, M.D.,3-6 Adnan H. Sidd iqui, M.D., Ph.D.,3-6 and Elad I. Levy, M.D.; Intracerebral hemorrhage secondary to intravenous and endovascular intraarterial revascularization therapies in acute ischemic stroke: an update on risk factors, predictors, and management. Neurosurg Focus 32 (4):E2, 2012
8. Maxim Mokin, M.D., Ph.D.,1 Alexander A. Khalessi, M.D., M.S.,3 J Mocco, M.D., M.S.,4 Giusepp e Lanzino, M.D.,5 Travis M. Dumont, M.D.,1 Ricardo A. Hanel, M.D., Ph.D.,6 Demetrius K. Lopes, M.D.,7 Richard D. Fessler II , M.D.,8 Andrew J. Ringer, M.D.,9 Bernard R. Bendok, M.D.,10 Erol Veznedaroglu, M.D.,11 Adnan H. Sidd iqui, M.D., Ph.D.,1,2 L. Nelson Hopkins, M.D.,1,2 and Elad I. Levy, M.D., M.B.A.1,2 ; Endovascular treatment of acute ischemic stroke: the end or just the beginning? ; Neurosurg Focus 36 (1):E5, 2014

9. Melanie G. Hayden, M.D., M.A.S., Marco Lee, M.D., Ph.D., F.R.C.S., Raph ael Guzman, M.D., and Gary K. Steinberg, M.D., Ph.D.; The evolution of cerebral revascularization surgery, Neurosurg Focus 26 (5):E17, 2009.

10. Raphael Guzman, M.D.,1,3 Marco Lee, M.D., Ph.D.,1,3 Achal Achrol, B.S.,1,3 Teresa Bell-Stephens, R.N.,1,3 Michael Kelly, M.D.,1,3 Huy M. Do, M.D.,1-3 Michael P. Marks , M.D.,1-3 and Gary K. Steinberg, M.D., Ph.D.1,3; Clinical outcome after 450 revascularization procedures for moyamoya disease; J Neurosurg 111:927-935, 2009

11. R. WEBSTER CROWLEY M.D.,1 RICKY MEDEL, M.D.,1 AND AARON S. DUMONT, M.D.1,2 Evolution of cerebral revascularization techniques; Neurosurg Focus 24 (2):E3, 2008

12. RICHARD LEBLANC, M.D., F.R.C.S.(C), JANE L. TYLER, M.D., G RARD MOHR, M.D., F.R.C.S.(C), ERNST MEYER, PH.D., MIRKO DIKSIC, PH.D., LUCAS YAMAMOTO, M.D., PH.D., LAUGHLIN TAYLOR, M.Sc., SERGE GAUTHIER, M.D., F.R.C.P.(C), AND ANTOINE HAKIM, M.D., PH.D., F.R.C.P.(C); Hemodynamic and metabolic effects of cerebral revascularization; J Neurosurg 66:529-535, 1987

13. Sunil Manjila, M.D.,1 Tony Masri, M.D.,2 Tanzila Shams, M.D.,2 Shakeel A. Chowdhry, M.D.,1 Cathy Sila, M.D.,2 and Warren R. Selman, M.D.1 ; Evidence-based review of primary and secondary ischemic stroke prevention in adults: a neurosurgical perspective; Neurosurg Focus 30 (6):E1, 2011

14. Sanai N, Zador Z, Lawton MT: Bypass surgery for complex brain aneurysms: an assessment of intracranial intracranial bypass, Neurosurgery 65: 670-683, 2009 